\title{
Distribuição geográfica e indicadores entomológicos de triatomíneos sinantrópicos capturados no Estado de Goiás
}

\author{
Geographical distribution and indicators entomologic of sinantropic \\ triatomines captured in the State of Goiás
}

\author{
Antônio Wilson Soares de Oliveira ${ }^{1,2}$ e Ionizete Garcia da Silva ${ }^{1,3}$
}

\begin{abstract}
RESUMO
Apresenta-se a distribuição geográfica de triatomíneos em Goiás e indicadores entomológicos no ambiente domiciliar: infestação domiciliar, densidade triatomínica domiciliar e infecção vetorial pelo Trypanosoma cruzi. Os indicadores estudados foram de triatomíneos capturados no intra e peridomicílio, em 201 municípios, no Estado de Goiás, Brasil, no perído entre 2000 e 2003. Foram investigadas 249.868 unidades domiciliares e capturados 51.570 triatomíneos, com 335 espécimes infectados com Trypanosoma cruzi. A infestação peridomiciliar foi significativamente maior do que a intradomiciliar na espécie Triatoma sordida, seguida de Panstrongylus megistus. O inverso ocorreu nas espécies Rhodnius neglectus, Panstrongylus geniculatus $e$ Triatoma pseudomaculata $(p<0,018)$. Não bouve diferença significativa entre as infestações intra e peridomiciliar nas espécies Panstrongylus diasi, Triatoma costalimai e Triatoma williami. Apenas um exemplar da espécie Triatoma infestans foi capturado no ano 2000
\end{abstract}

Palavras-chaves: Doença de Chagas. Trypanosoma cruzi. Triatoma sordida. Panstrongylus megistus. Triatoma infestans.

\begin{abstract}
The geographic distribution of triatomines in Goiás and entomological indicators in the home environment are presented: bome infestation, bome triatomine density and vector infection by Trypanosoma cruzi. The indicators studied were from triatomines captured inside and outside homes in 201 municipalities in the State of Goiás, Brazil, between 2000 and 2003. A total of 249,868 home units were investigated and 51,570 triatomines were captured, and 335 specimens were found to be infected with Trypanosoma cruzi. Infestation outside the home was significantly greater than infestation inside the bome for the species Triatoma sordida, followed by Panstrongylus megistus. The contrary was observed with the species Rhodnius neglectus, Panstrongylus geniculatus and Triatoma pseudomaculata $(p<0.018)$. There was no significant difference between infestations inside and outside the home for the species Panstrongylus diasi, Triatoma costalimai and Triatoma williami. Only one specimen of Triatoma infestans was captured in the year 2000.
\end{abstract}

Key-words: Chagas' disease. Trypanosoma cruzi. Triatoma sordida. Panstrongylus megistus. Triatoma infestans.

A doença de Chagas é a protozoose de maior importância epidemiológica no Estado de Goiás, Brasil. Classificada no meio científico como doença negligenciada, sua prevalência e distribuição estão intimamente relacionadas a fatores ambientais, sócio-culturais e políticos. A transmissão vetorial sempre esteve associada ao estreito contato do homem-triatomíneos, principalmente na zona rural, com infestação intradomiciliar. Dados do Sistema de Mortalidade (SIM-MS) mostram que a doença de Chagas foi responsável por 3.321 óbitos em Goiás, no período de 1999 a 2002, com maior ocorrência nas faixas etárias mais elevadas, cuja infecção possivelmente, se deu há vários anos atrás.

O Estado de Goiás recebeu em março de 2000, o certificado da Organização Pan-Americana da Saúde, considerando eliminado do seu território o Triatoma infestans, até então a principal espécie vetora da doença de Chagas na região. Este título reforça ainda mais a necessidade de se manter uma vigilância entomológica rotineira e sustentável em relação à reinfestação desse vetor, bem como a necessidade de monitorar o comportamento relativo

1. Pós-graduação em Medicina Tropical do Instituto de Patologia Tropical e Saúde Pública da Universidade Federal de Goiás, Goiânia, GO. 2. Superintendência de Políticas de Atenção Integral à Saúde da Secretaria de Estado da Saúde de Goiás. Goiânia, GO. 3. Laboratório de Biologia e Fisiologia de Insetos e Xenodiagnóstico do Instituto de Patologia Tropical e Saúde Pública da Universidade Federal de Goiás, Goiânia, GO.

Endereço para correspondência: Dr. Ionizete Garcia da Silva. Depto Microbiologia, Imunologia, Parasitologia e Patologia/IPTSP/ UFG. Caixa Postal 131, $74001-970$ Goiânia, G0. Tel: 5562 3209-6128; Fax: 5562 3261-2077

e-mail: ionizete@iptsp.ufg.br

Recebido para publicação em 23/5/2006

Aceito em 2/4/2007 
à domiciliação e capacidade de infecção de outras espécies silvestres que invadem as casas ${ }^{3811} 1215161819$.

Dados mais recentes sobre a distribuição geográfica e frequiência de triatomíneos no Estado de Goiás foram mostrados há uma década atrás $^{14}{ }^{16}$. 0 conhecimento atualizado da distribuição geográfica, infestação peri ou intradomiciliar e a infecção de triatomíneos por Trypanosoma cruzi são subsídios fundamentais para o planejamento das ações de controle e vigilância entomológica municipal. Além disso, é importante para o planejamento de prioridades das ações estaduais, financiamento, acompanhamento e supervisão do controle da doença de Chagas, afastando o risco de recrudescimento de triatomíneos nas áreas mais carentes do estado ${ }^{127}$.

\section{MATERIAL E MÉTODOS}

No período de 2000 a 2003, foram realizadas capturas de triatomíneos em 201 dos 246 municípios existentes no Estado de Goiás. A seleção dos municípios a serem trabalhados, no período delimitado para este estudo, foi feita pela Coordenação Estadual do Programa de Controle da Doença de Chagas (PCDCH) no Estado de Goiás, de acordo com critérios de prioridade epidemiológica e a capacidade instalada de cada município para realização das atividades programadas. Os espécimes foram capturados no ambiente intra e peridomiciliar por agentes de saúde em visitas domiciliares programadas anualmente sem utilização de desalojantes. Foi utilizadas pinças metálicas e lanternas de 2 ou 4 pilhas para inspeção em frestas e locais desprovidos de luminosidade que possam servir de abrigo para os triatomíneos. Os exemplares foram acondicionados em frascos de polietileno, com retalhos de papel dobrados em forma de "Z" para melhor preserválos. Recomendou-se o número máximo de 5 exemplares em cada frasco. Os frascos foram devidamente numerados, etiquetados e registrados em formulários específicos do $\mathrm{PCDCH}$.

Em seguida, os triatomíneos foram encaminhados aos laboratórios regionais para identificação taxonômica e exame da infecção por T. cruzi com base no manual de normas sobre organização e funcionamento de laboratórios de diagnostico da doença de Chagas $^{17}$.
A revisão amostral ficou a cargo do Laboratório de Biologia e Fisiologia de Insetos e Entomologia, do Instituto de Patologia Tropical e Saúde Pública (IPTSP) da Universidade Federal de Goiás (UFG) em parceria com a Fundação Nacional de Saúde (FUNASA) e Secretaria de Estado da Saúde de Goiás (SES-GO).

Os triatomíneos infectados por T.cruzi foram georreferenciados por localidade, por meio de coordenadas geográficas obtidas com o auxílio do Global Positon System (GPS).

Estudaram-se os seguintes indicadores entomológicos: infestação domiciliar (ID), densidade triatomínica domiciliar (DTD), infecção natural (IN) por T. cruzi, de acordo com orientação já estabelecida ${ }^{6}$. Os dados tiveram como base 0 sistema de informações do programa de controle da doença de Chagas instalados nos NACEs dos municípios de Ceres, Formosa, Jataí e Morrinhos. Nas análises estatísticas foram utilizados os testes qui-quadrado e teste exato de Fisher ao nível de significância de $5 \%$.

Indicadores entomológicos, conforme orientação preconizada pelo Ministério da Saúde ${ }^{6}$ e OPAS $^{9}$ :

\section{ID = Número de unidades domiciliares com presença de triatomíneos x100 \\ Número de unidades domiciliares pesquisadas}

$$
\text { DTD }=\frac{\text { Número de exemplares de triatomíneos capturados }}{\text { Número de unidades domiciliares pesquisadas }}
$$

$$
\text { IN = Número de triatomíneos infectados por T.cruzi } \times 100
$$$$
\text { Número de triatomíneos examinados }
$$

\section{RESULTADOS}

A investigação de 249.868 unidades domiciliares, no ambiente intra e peridomiciliar, resultou na captura de 51.570 espécimes de triatomíneos. Foram identificadas nove espécies de triatomíneos, das quais 335 exemplares estavam infectadas com T. cruzi (Tabelas 1 e 3). Comparando-se os indicadores de infestação intra e peridomiciliar de cada espécie, verificou-se uma

\begin{tabular}{|c|c|c|c|c|c|c|}
\hline & \multicolumn{2}{|c|}{ Intradomicílio } & \multicolumn{2}{|c|}{ Peridomicílio } & \multicolumn{2}{|c|}{ Total } \\
\hline & capturados & positivos & capturados & positivos & capturados & positivos \\
\hline Panstrongylus diasi & 6 & 0 & 12 & 0 & 18 & 0 \\
\hline Panstrongylus geniculatus & 142 & 2 & 63 & 0 & 205 & 2 \\
\hline Panstrongylus megistus & 376 & 2 & 1.066 & 5 & 1.442 & 7 \\
\hline Rhodnius neglectus & 2.051 & 31 & 2.499 & 16 & 4.550 & 47 \\
\hline Triatoma costalimai & 139 & 0 & 128 & 0 & 267 & 0 \\
\hline Triatoma infestans & 1 & 0 & 0 & 0 & 1 & 0 \\
\hline Triatoma pseudomaculata & 316 & 7 & 353 & 2 & 669 & 9 \\
\hline Triatoma sordida & 5.555 & 46 & 38.819 & 223 & 44.374 & 269 \\
\hline Triatoma williami & 19 & 1 & 25 & 0 & 44 & 1 \\
\hline Total & 8.605 & 89 & 42.965 & 246 & 51.570 & 335 \\
\hline
\end{tabular}

Tabela 1-Triatomíneos sinantrópicos capturados e positivos por Trypanosoma cruzi no Estado de Goiás, no período de 2000 a 2003. 
infestação peridomiciliar significativamente maior nas espécies T. sordida e P. megistus $(\mathrm{p}<0,001)$. A infestação intradomiciliar foi significativamente maior do que a peri nas espécies R. neglectus, P. geniculatus e T. pseudomaculata $(\mathrm{p}<0,018)$. Não houve diferença significativa nas infestações intra e peridomiciliar nas espécies $P$. diasi, T. costalimai e T. williami (Tabelas 2). No período estudado foi capturado apenas um exemplar da espécie T. infestans, no intradomicílio, no ano 2000 (Figura 1).

Na comparação entre as espécies, verificou-se que T. sordida apresentou indicadores de infestação e densidade no intradomicílio significativamente maiores do que o somatório de todas as demais espécies estudadas $(\mathrm{p}<0,001)$. A segunda espécie mais densa no intradomicílio foi $R$. neglectus, sendo significativamente maior do que o somatório das demais encontradas $(\mathrm{p}<0,001)$. Em relação ao indicador de infecção natural (IN) apenas a espécie R. neglectus apresentou diferença significativa entre os valores encontrados no intra e peridomicílio, sendo significativamente maior no intradomicílio, com $\mathrm{p}<0,001$ (Tabela 4).

Em relação à distribuição geográfica a espécie T. sordida foi a que apresentou maior dispersão, sendo encontrada em 79,6\% dos municípios, destes $31,3 \%$ encontraram-se triatomíneos infectados por T. cruzi (Tabela 3). A espécie R. neglectus foi encontrada em
Tabela 2 - Infestação de triatomíneos sinantrópicos no Estado de Goiás, no período de 2000 a 2003.

\begin{tabular}{llcrr}
\hline Espécies & Intradomicílio (I) & Peridomicilio (P) & $\mathrm{X}^{2}(\mathrm{I} \times \mathrm{P})$ & \multicolumn{1}{c}{$\mathrm{p}$} \\
\hline Panstrongylus diasi & 0,001 & 0,003 & 0,90 & 0,344 \\
Panstrongylus geniculatus & 0,04 & 0,01 & 39,62 & $<0,001$ \\
Panstrongylus megistus & 0,13 & 0,33 & 235,56 & $<0,001$ \\
Rhodnius neglectus & 0,61 & 0,47 & 45,12 & $<0,001$ \\
Triatoma costalimai & 0,02 & 0,03 & 1,15 & $<0,283$ \\
Triatoma pseudomaculata & 0,06 & 0,04 & 5,61 & 0,018 \\
Triatoma sordida & 1,60 & 8,99 & $1.3596,29$ & $<0,001$ \\
Triatoma williami & 0,004 & 0,004 & 0,00 & 1,000 \\
\hline
\end{tabular}

Tabela 3 - Densidade de triatomíneos sinantrópicos por unidade domiciliar no Estado de Goiás, no período de 2000 a 2003.

\begin{tabular}{lccrc}
\hline Espécies & Intradomicílio (I) & Peridomicilio (P) & $\mathrm{X}^{2}(\mathrm{I} \times \mathrm{P})$ & $\mathrm{p}$ \\
\hline Panstrongylus diasi & 0,00002 & 0,00005 & 1,39 & 0,2386 \\
Panstrongylus geniculatus & 0,0006 & 0,0003 & 29,69 & $<0,001$ \\
Panstrongylus megistus & 0,0015 & 0,0043 & 330,16 & $<0,001$ \\
Rhodnius neglectus & 0,0082 & 0,01 & 44,32 & $<0,001$ \\
Triatoma costalimai & 0,0006 & 0,0005 & 0,37 & 0,5400 \\
Triatoma pseudomaculata & 0,0013 & 0,0014 & 1,94 & 0,1637 \\
Triatoma sordida & 0,0222 & 0,1554 & 27363,91 & $<0,001$ \\
Triatoma williami & 0,0001 & 0,0001 & 0,57 & 0,4451 \\
\hline
\end{tabular}

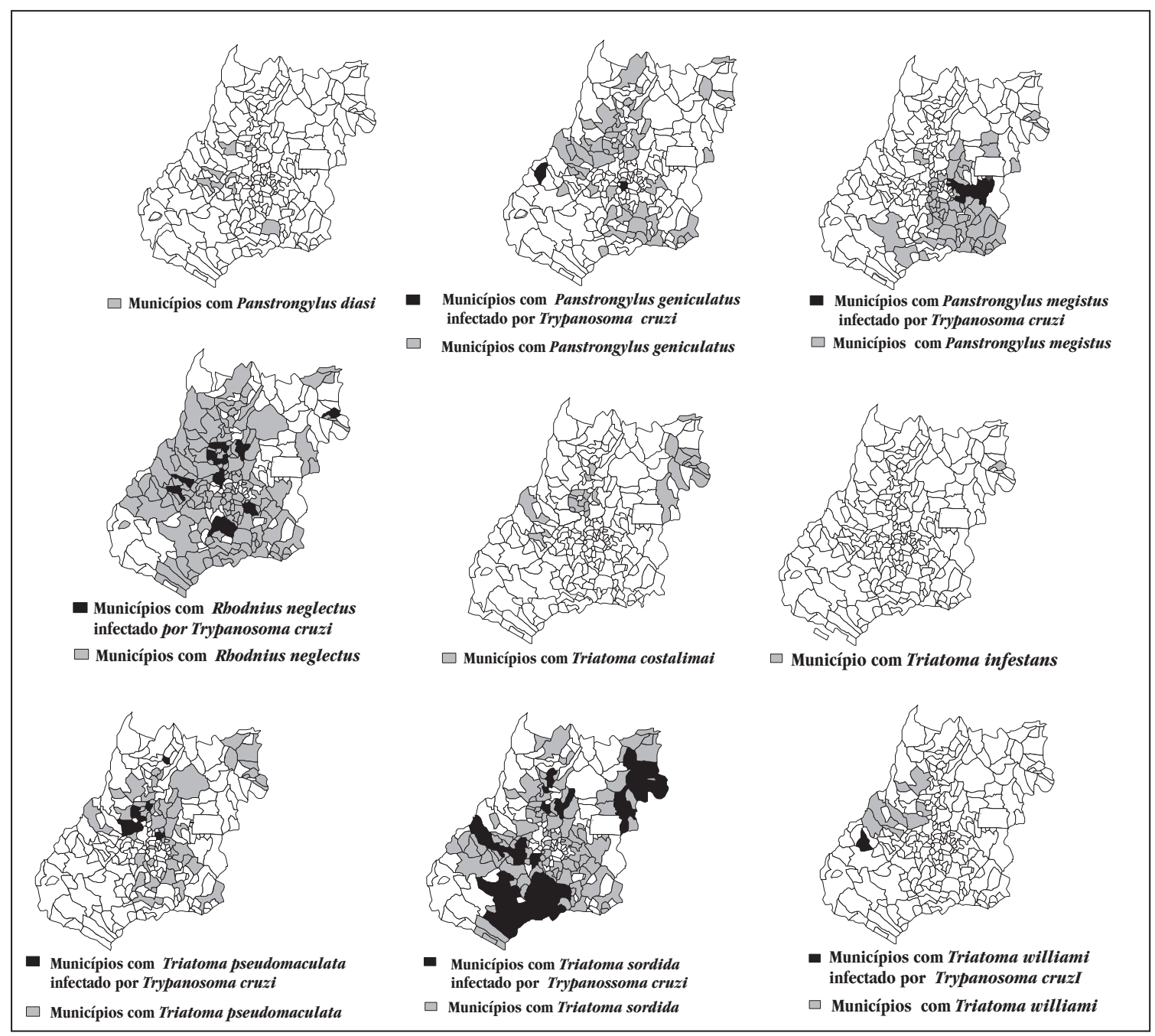

Figura 1 - Distribuição geográfica de espécies de triatomíneos sinantrópicos e freqüiência de positividade para Trypanosoma cruzi, por município, Goiás, Brasil, 2000-2003. 
Tabela 4 - Percentagem da infecção natural por Trypanosoma cruzi em triatomíneos sinantrópicos no Estado de Goiás, no período de 2000 a 2003.

\begin{tabular}{lcccc}
\hline Espécies & Intradomicílio (I) & Peridomicilio (P) & $\mathrm{X}^{2}(\mathrm{IxP})$ & $\begin{array}{c}\text { Teste de Fisher (IXP) } \\
\text { Panstrongylus diasi }\end{array}$ \\
Panstrongylus geniculatus & 0,000 & 0,000 & - & - \\
Panstrongylus megistus & 1,418 & 0,000 & & 0,495 \\
Rhodnius neglectus & 0,548 & 0,469 & & 0,745 \\
Triatoma costalimai & 1,579 & 0,681 & 7,20 & 0,007 \\
Triatoma pseudomaculata & 0,000 & 0,000 & - & - \\
Triatoma sordida & 2,215 & 0,571 & & 0,093 \\
Triatoma williami & 0,839 & 0,620 & 3,120 & 0,077 \\
\hline
\end{tabular}

79,1\% dos municípios, dos quais 9,4\% estavam infectados pelo Trypanosoma cruzi.

\section{DISCUSSÃo}

Com a eliminação do T. infestans, as espécies T. sordida e $R$. neglectus se destacam, atualmente, como as mais importantes, epidemiologicamente, no Estado de Goiás. Isso se deve não só aos indicadores entomológicos de infestação e densidade domiciliar, significativamente maiores que as demais, mas por estarem presentes na maioria dos municípios do Estado de Goiás.

Comparando todas as espécies estudadas, T. sordida foi a que apresentou os maiores indicadores de infestação e densidade, mostrando preferência pelo ambiente peridomiciliar, provavelmente pelo comportamento ornitofílico da espécie, como mencionado em outros estudos ${ }^{1014}$.

Com exceção de T. sordida, a espécie $R$. neglectus mostrou indicadores de infestação e densidade superiores às demais espécies. Na comparação dos indicadores do peri e intradomicílio, $R$. neglectus evidenciou uma significativa infestação intradomiciliar, o que não pode ser concebido como uma preferência por este ambiente já que a densidade dessa espécie foi significativamente maior no peridomicílio. Esta situação também foi observada por outros autores ${ }^{4}{ }^{16}$, que alertam sobre a extensa distribuição geográfica e a constante presença peridomiciliar, aumentando a possibilidade dessa espécie adaptar-se ao ambiente domiciliar.

Foi capturado e identificado apenas um exemplar de Triatoma infestans no intradomicílio, no ano 2000, corroborando o certificado de eliminação da espécie no Estado de Goiás. A redução da frequiência do T. infestans no Estado de Goiás já tinha sido observada ${ }^{13}{ }^{14}$, comparando capturas realizadas entre 1984/1988 e 1992/1993. Este fato está associado às ações de controle desenvolvidas ${ }^{516}$ ao longo do tempo, que se mostraram eficazes para o T. infestans, por ser uma espécie alóctone.

A distribuição geográfica no Estado de Goiás, das espécies T. infestans e T. sordida deste trabalho é semelhante à verificada por Silveira ${ }^{16}$, no período de 1975 a 1983 , contudo, tenha sido evidenciado também, uma dispersão dessas espécies e a presença de outras no ambiente domiciliar, como T. costalimai $\mathrm{e}$ Triatoma williami.
Embora o Estado de Goiás tenha recebido o certificado de eliminação do T. infestans, principal espécie vetora em anos anteriores, a captura de outras espécies, infectadas pelo T. cruzi no ambiente intradomiciliar é motivo de preocupação e alerta. Recomenda-se manter nos municípios um trabalho rotineiro e eficiente de vigilância entomológica capaz de detectar mudanças de comportamento das atuais espécies, bem como evitar uma reinfestação do T. infestans, espécie que ainda não foi eliminada do Estado da Bahia, que faz limite com a região do nordeste goiano. Outro ponto fundamental está relacionado à necessidade da implementação de ações integradas entre os serviços de saúde e os centros de pesquisa locados nas instituições de ensino superior que permitam o desenvolvimento de pesquisas, contribuindo na orientação da vigilância entomológica, principalmente em relação ao comportamento e controle das espécies de triatomíneos que invadem e estabelecem colônias nas moradias em vários municípios de Goiás.

\section{AGRADECIMENTOS}

Aos chefes e equipes dos Núcleos de Apoio ao Controle de Endemias da Secretaria de Estado da Saúde de Goiás pelo imprescindível apoio nos trabalhos de campo. À Dr ${ }^{\mathrm{a}}$ Elizabeth Carmen Duarte pelas contribuições nas análises estatísticas.

\section{REFERÊNCIAS}

1. Dias JCP. Vigilância epidemiológica em doença de Chagas. Caderno de Saúde Pública 16:43-59, 2000.

2. Dias JCP. Doença de Chagas, participação e Estado. Caderno de Saúde Pública 17:165-169, 2001.

3. Elias CN, Silva IG, Camargo MF, Alves RBN. Índice de infecção de triatomíneos pelo Trypanosoma cruzi capturados no ambiente domiciliar no Estado de Goiás em 1993. Revista de Patologia Tropical 23:169-174, 1994.

4. Forattini PF, Rabello EX, Ferreira OA, Silva ERS, Santos JLF. Aspectos ecológicos da tripanossomíase americana. XXI - Comportamento de espécies triatomíneos silvestres na reinfestação do intra e peridomicílio. Revista de Saúde Pública 18:185-208, 1984.

5. Fundação Nacional de Saúde. Controle da doença de Chagas. Diretrizes técnicas. Ministério da Saúde, Brasília, 1994.

6. Lenti H, Wygodzinsky P. Revision of triatominae (Hemiptera, Reduviidae), and their significance as vectors of Chagas disease. Bolletin of the American Museum of Natrural History 163:125-520, 1979. 
7. Linhares CV. Vetores do Trypanosoma cruzi. Revista de Patologia Tropical 29:83-89, 2000.

8. Oliveira AWS, Sisteroli GC, Albernaz VGP, Marinho FE. Triatoma infestans no Estado de Goiás. Revista da Sociedade Brasileira de Medicina Tropical 36:395-396, 2003.

9. Organización Panamericana de la Salud. Guía para muestreo en actividades de vigilancia y control vectorial de la enfermedad de Chagas, 2003.

10. Pires HHR, Borges ECB, Andrade REA, Lorosa ES, Diotaiuti L. Peridomiciliary Triatoma sordida Stal,1859 inthe County of Serra do Ramalho, Bahia, Brazil. Memórias do Instituto Osvaldo Cruz 94:147-149, 1999.

11. Rassi A, Luquetti A0, Ornelas JF. Impacto do controle químico extensivo de Triatoma infestans sobre a incidência de casos agudos e a prevalência de doença de Chagas: 0 exemplo de Montalvânia, Minas Gerais. Revista da Sociedade Brasileira Medicina Tropical 36:719-727, 2003

12. Schmunis GA, Dias JCP. Health system decentralization, and the control of vector-borne diseases. Cadernos de Saúde Pública 16:117-123, 2000.

13. Silva IG, Silva JL, Silva HHG, Camargo MF, Moura AF, Santos AH. Distribuição dos vetores da tripanossomíase americana capturados no ambiente domiciliar no Estado de Goiás. Anais da Sociedade Entomológica do Brasil 21:140-154, 1992 .

14. Silva IG, Silva JL, Camargo MF, Elias CN, Santos AH, Silva HHG, Isac E, Alves RBN Infestação de vetores da tripanossomíase americana no ambiente domiciliar no Estado de Goiás. Revista de Patologia Tropical 24:41-47, 1995.

15. Silveira AC. Situação do controle da doença de Chagas nas Américas. Cadernos de Saúde Pública 16:35-42, 2000.

16. Silveira AC, Feitosa VR, Borges R. Distribuição de triatomíneos capturados no ambiente domiciliar, no período de 1975/83, Brasil. Revista Brasileira de Malariologia e Doenças Tropicais 36: 15-312, 1984

17. Superintendência de Campanhas de Saúde Pública. Manual de normas sobre organização e funcionamento de laboratórios de diagnóstico da doença de Chagas, Ministério da Saúde, Brasília, 1981.

18. Telleria AV. Situação da doença de Chagas no Continente Sul-Americano. Revista de Patologia Tropical 29:75-82, 2000.

19. Vinhaes MC, Dias JCP. Doença de Chagas no Brasil. Cadernos de Saúde Pública 16:7-12, 2000. 